\title{
On a Multiplicative Partition Function
}

\author{
Yifan Yang \\ Department of Mathematics \\ University of Illinois
}

Submitted: October 5, 2000; Accepted: April 12, 2000

MR Subject Classification: primary 11N60, secondary 05A18, 11P82

\begin{abstract}
Let $D(s)=\sum_{m=1}^{\infty} a_{m} m^{-s}$ be the Dirichlet series generated by the infinite product $\prod_{k=2}^{\infty}\left(1-k^{-s}\right)$. The value of $a_{m}$ for squarefree integers $m$ with $n$ prime factors depends only on the number $n$, and we let $f(n)$ denote this value. We prove an asymptotic estimate for $f(n)$ which allows us to solve several problems raised in a recent paper by M. V. Subbarao and A. Verma.
\end{abstract}

\section{Introduction and Statements of Results}

Let $D(s)=\sum_{m=1}^{\infty} a_{m} m^{-s}$ be the Dirichlet series generated by the infinite product $\prod_{k=2}^{\infty}\left(1-k^{-s}\right)$. The coefficients $a_{m}$ denote the excess of the number of (unordered) representations of $m$ as a product of an even number of distinct integers $>1$ over the number of representation of $m$ as a product of an odd number of distinct integers $>1$. The Dirichlet series $D(s)$ is closely related to the generating Dirichlet series in the "Factorisatio Numerorum" problem of Oppenheim (see [6]). Indeed, if we let $b_{m}$ denote the number of (unordered) representations of $m$ as a product of integers $>1$, not necessarily distinct, then we have $\sum_{m=1}^{\infty} b_{m} m^{-s}=D(s)^{-1}$. Thus, by the Möbius inversion formula, the numbers $a_{m}$ and $b_{m}$ are related by the identity $a_{m}=\sum_{d \mid m} \mu(d) b_{m / d}$. Oppenheim [6] showed that

$$
\frac{1}{x} \sum_{m \leq x} b_{m} \sim \frac{e^{\sqrt{\log x}}}{2 \sqrt{\pi}(\log x)^{3 / 4}}
$$

In [3], E. R. Canfield, P. Erdős and C. Pomerance proved that if $m$ is an integer such that $b_{n}<b_{m}$ for all $n<m$, then

$$
b_{m}=m \exp \left\{-(1+o(1)) \log m \log _{3} m / \log _{2} m\right\},
$$

where $\log _{k}$ denotes the $k$-times iterated logarithm.

In this paper, we consider the more difficult problem of investigating the asymptotic behavior of the numbers $a_{m}$. This problem was raised by M. V. Subbarao, who observed 
that $a_{m}=0, \pm 1$ for all positive integers $m$ with at most four prime factors and asked whether this is true for all $m$. It is easy to see that for a positive integer $m>1$ the coefficient $a_{m}$ depends only on the exponents $r_{1}, \ldots, r_{n}$ in the canonical prime factorization $m=p_{1}^{r_{1}} \ldots p_{n}^{r_{n}}$. In particular, for squarefree $m=p_{1} \ldots p_{n}$, the value of $a_{m}$ is a function of the number $n$ of prime factors of $m$. We will denote this function by $f(n)$.

The function $f(n)$ can be interpreted as a set-partition function. Indeed, by identifying factors of $m=p_{1} \ldots p_{n}$ with subsets of $\{1,2, \ldots, n\}$, we see that $f(n)$ is equal to the excess of the number of ways to partition a set $S$ of $n$ elements into an even number of non-empty subsets over the number of ways to partition $S$ into an odd number of non-empty subsets. Therefore, $f(n)$ can also be written as

$$
f(n)=\sum_{k=1}^{n}(-1)^{k} S_{2}(n, k)
$$

where the numbers $S_{2}(n, k)$ are the Stirling numbers of the second kind, which denote the number of partitions of an $n$-element set into $k$ non-empty subsets (see, e.g., [8, Section 3.6]).

A further motivation for studying the function $f(n)$ is the following observation of $\mathrm{D}$. Bowman [2]. For each integer $n>0$ there exist exactly one integer $b_{n}$ and a polynomial $P_{n}(x, y)$ such that

$$
\sum_{k=0}^{m}\left(k^{n-1}+b_{n}\right) k !=P_{n}(m !, m)
$$

holds for all integers $m$. It turns out that this integer $b_{n}$ is equal to $f(n)$. By a simple proof by induction, we have $\sum_{k=0}^{m} k \cdot k !=(m+1) !-1$, and hence $f(2)=b_{2}=0$. The case $n=2$ is the only known case with $b_{n}=0$. H. S. Wilf raised the question whether $b_{n}=0$ (or equivalently $f(n)=0$ ) infinitely often.

By (1) we have the trivial upper bound

$$
|f(n)| \leq \sum_{k=1}^{n} S_{2}(n, k)
$$

The numbers $B(n)=\sum_{k=1}^{n} S_{2}(n, k)$ are known as Bell numbers (see, e.g., [8, Section 1.6]). De Bruijn [4] gave a detailed asymptotic analysis of $B(n)$, using the saddle point method. In particular, de Bruijn [4, p. 108] showed that

$$
\log B(n)=n\left(L-L_{2}-1+\frac{L_{2}+1}{L}+\frac{L_{2}^{2}}{2 L^{2}}+O\left(\frac{L_{2}^{3}}{L^{3}}\right)\right),
$$

where $L=\log n$ and $L_{2}=\log \log n$. Therefore we have the upper bound

$$
\limsup _{n \rightarrow \infty} \frac{\log |f(n)|}{n \log n} \leq 1
$$

In a recent paper Subbarao and A. Verma [7] showed that in fact

$$
\limsup _{n \rightarrow \infty} \frac{\log |f(n)|}{n \log n}=1 .
$$


Thus the coefficients $a_{m}$ in the Dirichlet series $\sum_{m=1}^{\infty} a_{m} m^{-s}=\prod_{k=2}^{\infty}\left(1-k^{-s}\right)$ are not uniformly bounded. This answers the question of Subbarao mentioned earlier. (This result was also obtained by P. T. Bateman [1].)

In this paper we provide a detailed asymptotic analysis of $f(n)$, which allows us to answer some open problems mentioned in [7]. Our main result is the following theorem, which gives an asymptotic estimate for $f(n)$.

Theorem 1 Let $z_{n}$ be the solution to the equation $z e^{z}=-n-1$ with the smallest positive imaginary part. Let $\phi_{n}(z)=-e^{z}-(n+1) \log z$, and let $w_{n}$ be the solution of $w_{n}^{2}=$ $-2 / \phi_{n}^{\prime \prime}\left(z_{n}\right)$ with $\pi / 2<\arg w_{n}<\pi$. Then we have

$$
f(n)=\operatorname{Im} \Phi(n)+O\left(\frac{\log n}{n}|\Phi(n)|\right),
$$

where

$$
\Phi(n)=\frac{n ! e}{\sqrt{\pi}} w_{n} \exp \left\{\phi_{n}\left(z_{n}\right)\right\}
$$

Using estimates for $z_{n}$ and $w_{n}$ (see Lemma 1 below), we obtain the following asymptotic upper bound for $\log |f(n)|$, which sharpens (3). We recall here the notations

$$
L=\log n, \quad L_{2}=\log \log n
$$

introduced earlier.

Corollary 1 We have, for $n \geq 3$,

$$
\log |f(n)| \leq n\left(L-L_{2}-1+\frac{L_{2}+1}{L}+\frac{L_{2}^{2}-\pi^{2}}{2 L^{2}}+O\left(\frac{L_{2}^{3}}{L^{3}}\right)\right) .
$$

Comparing this bound with the estimate (2) for the Bell numbers $B(n)$, we obtain the following corollary, which shows the cancellation effect occuring in the sum $f(n)=$ $\sum_{k=1}^{n}(-1)^{k} S_{2}(n, k)$, when compared to $B(n)=\sum_{k=1}^{n} S_{2}(n, k)$.

Corollary 2 We have, for $n \geq 3$,

$$
\log |f(n)| \leq \log B(n)-\frac{\pi^{2} n}{2 L^{2}}+O\left(\frac{n L_{2}^{3}}{L^{3}}\right) .
$$

By investigating the behavior of the argument of $\log \Phi(n)$, we can determine how often $f(n)$ changes signs. This is the content of the following two corollaries.

Corollary 3 Let $\Phi(n)$ be defined as in Theorem 1. Then we have

$$
f(n)=|\Phi(n)|\left(\sin \theta(n)+O\left(\frac{\log n}{n}\right)\right),
$$


where $\theta(t)$ is a differentiable function defined on $[3, \infty)$ satisfying

$$
\begin{aligned}
& \theta(t)=-\frac{\pi t}{\log t}+O\left(\frac{t \log \log t}{(\log t)^{2}}\right), \\
& \theta^{\prime}(t)=-\frac{\pi}{\log t}+O\left(\frac{\log \log t}{(\log t)^{2}}\right),
\end{aligned}
$$

and

$$
\theta^{\prime \prime}(t)=\frac{\pi}{t(\log t)^{2}}+O\left(\frac{\log \log t}{t(\log t)^{3}}\right)
$$

This result shows that $f(n)$ changes signs infinitely often and that $|f(n)|$ is not eventually monotone. This answers two questions raised by Subbarao and Verma [7].

The following result gives a precise estimate for the locations of the sign changes of $f(n)$.

Corollary 4 Let $n_{1}<n_{2}<\ldots$ denote the sequence of integers at which $f(n)$ changes signs, i.e., at which $f\left(n_{k}\right) \leq 0<f\left(n_{k}+1\right)$ or $f\left(n_{k}\right) \geq 0>f\left(n_{k}+1\right)$. Then

$$
n_{k}=k \log k+O(k \log \log k)
$$

and

$$
n_{k+1}-n_{k}=\log k+O(\log \log k) .
$$

Corollary 4 implies that the density of zeros of $f(n)$ is zero. In particular, we have

$$
|\{n \leq x: f(n)=0\}| \ll \frac{x}{\log x} .
$$

However, by a different approach, we can improve this bound.

Theorem 2 We have

$$
|\{n \leq x: f(n)=0\}| \ll x^{2 / 3}
$$

This result provides a partial answer to the question mentioned above whether $f(n)=0$ infinitely often.

To prove Theorem 1, we adapt the approach used by de Bruijn [4] to study the behavior of $B(n)$. We then use exponential sum estimates to prove Theorem 2 .

\section{Proof of Theorem 1}

In this section we continue to use the notations $L, L_{2}$ given in (4). We first deduce some useful estimates for the quantities $z_{n}, w_{n}$ and $\phi_{n}\left(z_{n}\right)$ defined in the statement of Theorem 1. 
Lemma 1 Let $z_{n}, w_{n}$ and $\phi_{n}(z)$ be defined as in the statement of Theorem 1 . Then we have

$$
\begin{gathered}
z_{n}=L-L_{2}+\pi i+\frac{L_{2}}{L}-\frac{\pi i}{L}+O\left(\frac{L_{2}^{2}}{L^{2}}\right), \\
w_{n}=\sqrt{\frac{2 L}{n}}\left(-\frac{\pi}{2 L}+i-\frac{i L_{2}}{2 L}-\frac{i}{2 L}+O\left(\frac{L_{2}^{2}}{L^{2}}\right)\right), \\
\phi_{n}\left(z_{n}\right)=n\left(-L_{2}+\frac{L_{2}+1}{L}-\frac{\pi i}{L}+\frac{L_{2}^{2}-\pi^{2}}{2 L^{2}}-\frac{\pi i L_{2}}{L^{2}}+O\left(\frac{L_{2}^{3}}{L^{3}}\right)\right) .
\end{gathered}
$$

Proof. By the definition of $z_{n}$, we have $e^{z_{n}}=-(n+1) / z_{n}$. This implies $\left|z_{n}\right| \ll L$, and by iteration we obtain

$$
\begin{aligned}
z_{n} & =\log (n+1)-\log z_{n}+\pi i \\
& =L+\pi i-\log \left(L-\log z_{n}+\pi i\right)+O\left(\frac{1}{n}\right) \\
& =L-L_{2}+\pi i+\frac{\log z_{n}}{L}-\frac{\pi i}{L}+O\left(\frac{L_{2}^{2}}{L^{2}}\right) \\
& =L-L_{2}+\pi i+\frac{L_{2}}{L}-\frac{\pi i}{L}+O\left(\frac{L_{2}^{2}}{L^{2}}\right) .
\end{aligned}
$$

This proves estimate (10).

Similarly, since $\phi_{n}^{\prime \prime}(z)=-e^{z}+(n+1) / z^{2}$ and thus $\phi_{n}^{\prime \prime}\left(z_{n}\right)=(n+1) / z_{n}+(n+1) / z_{n}^{2}$, we have, by (10),

$$
\begin{aligned}
w_{n}^{2} & =-\frac{2}{\phi_{n}^{\prime \prime}\left(z_{n}\right)}=-\frac{2 z_{n}}{n+1}\left(1+\frac{1}{z_{n}}\right)^{-1} \\
& =-\frac{2 L}{n}\left(1-\frac{L_{2}}{L}+\frac{\pi i}{L}+O\left(\frac{L_{2}}{L^{2}}\right)\right)\left(1-\frac{1}{L}+O\left(\frac{L_{2}}{L^{2}}\right)\right) .
\end{aligned}
$$

We then recall that, by the definition of $w_{n}, \pi / 2<\arg w_{n}<\pi$. Therefore

$$
\begin{aligned}
w_{n} & =i \sqrt{\frac{2 L}{n}}\left(1-\frac{L_{2}}{2 L}+\frac{\pi i}{2 L}+O\left(\frac{L_{2}^{2}}{L^{2}}\right)\right)\left(1-\frac{1}{2 L}+O\left(\frac{L_{2}}{L^{2}}\right)\right) \\
& =\sqrt{\frac{2 L}{n}}\left(-\frac{\pi}{2 L}+i-\frac{i L_{2}}{2 L}-\frac{i}{2 L}+O\left(\frac{L_{2}^{2}}{L^{2}}\right)\right),
\end{aligned}
$$

which is the claimed estimate (11). It remains to prove the estimate (12) for $\phi_{n}\left(z_{n}\right)$. 
By (10) and the definitions of $\phi_{n}(z)$ and $z_{n}$, we have

$$
\begin{aligned}
\phi_{n}\left(z_{n}\right)= & -e^{z_{n}}-(n+1) \log z_{n}=\frac{n+1}{z_{n}}-(n+1) \log z_{n} \\
= & \frac{n}{L-L_{2}+\pi i+\frac{L_{2}}{L}-\frac{\pi i}{L}+O\left(\frac{L_{2}^{2}}{L^{2}}\right)} \\
& -n \log \left(L-L_{2}+\pi i+\frac{L_{2}}{L}-\frac{\pi i}{L}+O\left(\frac{L_{2}^{2}}{L^{2}}\right)\right)+O\left(L_{2}\right) \\
= & n\left(\frac{1}{L}+\frac{L_{2}}{L^{2}}-\frac{\pi i}{L^{2}}+O\left(\frac{L_{2}^{2}}{L^{3}}\right)\right) \\
& -n\left(L_{2}-\frac{L_{2}}{L}+\frac{\pi i}{L}+\frac{L_{2}}{L^{2}}-\frac{\pi i}{L^{2}}-\frac{1}{2 L^{2}}\left(L_{2}-\pi i\right)^{2}+O\left(\frac{L_{2}^{3}}{L^{3}}\right)\right) \\
= & n\left(-L_{2}+\frac{L_{2}+1}{L}-\frac{\pi i}{L}+\frac{L_{2}^{2}-\pi^{2}}{2 L^{2}}-\frac{\pi i L_{2}}{L^{2}}+O\left(\frac{L_{2}^{3}}{L^{3}}\right)\right) .
\end{aligned}
$$

This proves (12) and completes the proof of the lemma.

Proof of Theorem 1. By the definition of $f(n)$, we have

$$
f(n)=\sum_{\substack{0<n_{1}<\ldots<n_{r} \\ a_{1}, \ldots, a_{r}>0 \\ a_{1} n_{1}+\cdots+a_{r} n_{r}=n}} \frac{(-1)^{a_{1}+\cdots+a_{r}} n !}{a_{1} ! \ldots a_{r} !\left(n_{1} !\right)^{a_{1}} \ldots\left(n_{r} !\right)^{a_{r}}} .
$$

Thus the exponential generating function for $f(n)$ is given by

$$
\begin{aligned}
\sum_{n=0}^{\infty} \frac{f(n)}{n !} z^{n} & =\sum_{\substack{n_{1}<\ldots<n_{r} \\
a_{1}, \ldots, a_{r}>0}} \frac{(-1)^{a_{1}+\cdots+a_{r}} z^{a_{1} n_{1}+\cdots+a_{r} n_{r}}}{a_{1} ! \ldots a_{r} !\left(n_{1} !\right)^{a_{1}} \ldots\left(n_{r} !\right)^{a_{r}}} \\
& =\prod_{n=1}^{\infty}\left\{\sum_{a=0}^{\infty} \frac{(-1)^{a}}{a !}\left(\frac{z^{n}}{n !}\right)^{a}\right\} \\
& =\exp (-z) \exp \left(-\frac{z^{2}}{2 !}\right) \exp \left(-\frac{z^{3}}{3 !}\right) \ldots \\
& =\exp \left\{-\left(e^{z}-1\right)\right\} .
\end{aligned}
$$

(For an alternative derivation of this identity see [7].) Using this generating function and Cauchy's formula, we obtain

$$
\frac{f(n)}{n ! e}=\frac{1}{2 \pi i} \int_{\mathcal{C}} \exp \left(-e^{z}\right) z^{-n-1} d z
$$

where $\mathcal{C}$ is a simple closed curve encircling the origin. Since $\exp \left(-e^{z}\right)$ is uniformly bounded in any half-plane $\{z: \operatorname{Re} z \leq \sigma\}$, the integration path $\mathcal{C}$ can be replaced by $\Gamma_{1} \cup \Gamma_{2}$, where 
$\Gamma_{1}=\left\{z_{n}+w_{n} t:-\operatorname{Im} z_{n} / \operatorname{Im} w_{n} \leq t<\infty\right\}$ and $\Gamma_{2}=\left\{\bar{z}_{n}-\bar{w}_{n} t:-\infty<t<\operatorname{Im} z_{n} / \operatorname{Im} w_{n}\right\}$, i.e., $\Gamma_{1}$ is the straight line lying in the upper half-plane that passes through $z_{n}$ in direction $w_{n}$, and the path $\Gamma_{2}$ is the reflection of $\Gamma_{1}$ with respect to the real axis, with direction $-\bar{w}_{n}$.

We now estimate the integral along $\Gamma_{1}$. Setting $z=z_{n}+w_{n} t$, we obtain

$$
\begin{aligned}
& \frac{1}{2 \pi i} \int_{\Gamma_{1}} \exp \left(-e^{z}\right) z^{-n-1} d z \\
= & \frac{w_{n}}{2 \pi i} \int_{-\operatorname{Im} z_{n} / \operatorname{Im} w_{n}}^{\infty} \exp \left\{\phi_{n}\left(z_{n}+w_{n} t\right)\right\} d t \\
= & \frac{w_{n} \exp \left\{\phi_{n}\left(z_{n}\right)\right\}}{2 \pi i}\left\{\int_{-\operatorname{Im} z_{n} / \operatorname{Im} w_{n}}^{-1 /\left|w_{n}\right|^{1 / 3}}+\int_{-1 /\left|w_{n}\right|^{1 / 3}}^{1 /\left|w_{n}\right|^{1 / 3}}+\int_{1 /\left|w_{n}\right|^{1 / 3}}^{\left|z_{n} / w_{n}\right|}+\int_{\left|z_{n} / w_{n}\right|}^{\infty}\right\} \\
& \quad \frac{w_{n} \exp \left\{\phi_{n}\left(z_{n}\right)\right\}}{2 \pi i}\left\{\phi_{n}\left(z_{n}+w_{n} t\right)-\phi_{n}\left(z_{n}\right)\right\} d t
\end{aligned}
$$

By estimates (10) and (11) of Lemma 1, we have, for $t \geq\left|z_{n} / w_{n}\right|$,

$$
\operatorname{Re} w_{n} t \leq\left(-\frac{\pi}{2 L}+O\left(\frac{L_{2}}{L^{2}}\right)\right)\left(L+O\left(L_{2}\right)\right)=-\frac{\pi}{2}+O\left(\frac{L_{2}}{L}\right),
$$

and thus

$$
\begin{aligned}
\operatorname{Re}\left(e^{z_{n}}-e^{z_{n}+w_{n} t}\right) & \leq-\operatorname{Re}\left(\frac{n+1}{z_{n}}\right)+e^{\operatorname{Re}\left(z_{n}+w_{n} t\right)} \\
& \leq-\left(1-e^{-\pi / 2}\right) \frac{n}{L}\left(1+O\left(\frac{L_{2}}{L}\right)\right) .
\end{aligned}
$$

Furthermore, since, by the same lemma,

$$
\arg w_{n}-\arg z_{n}=\frac{\pi}{2}+O\left(\frac{L_{2}}{L}\right),
$$

we have $\left|z_{n}+w_{n} t\right| \geq\left|w_{n} t\right|$ for sufficiently large $n$ and $t \geq\left|z_{n} / w_{n}\right|$. Using (13), it follows that

$$
\begin{aligned}
I_{4} & \leq \int_{\left|z_{n} / w_{n}\right|}^{\infty} \exp \left\{\operatorname{Re}\left(e^{z_{n}}-e^{z_{n}+w_{n} t}-(n+1) \log \left|\frac{z_{n}+w_{n} t}{z_{n}}\right|\right)\right\} d t \\
& \leq \int_{\left|z_{n} / w_{n}\right|}^{\infty} \exp \left\{-\frac{c_{1} n}{L}-n \log \left(\frac{\left|w_{n}\right|}{\left|z_{n}\right|} t\right)\right\} d t \\
& =\frac{\left|z_{n}\right|}{(n-1)\left|w_{n}\right|} \exp \left\{-\frac{c_{1} n}{L}\right\} \ll \sqrt{\frac{n}{L^{3}}} \exp \left\{-\frac{c_{1} n}{L}\right\}
\end{aligned}
$$

for sufficiently large $n$, where $c_{1}$ is a suitable positive constant. 
We next estimate $I_{3}$. We first show that $\operatorname{Re}\left(e^{z_{n}}-e^{z_{n}+w_{n} t}\right) \ll t \sqrt{n / L^{3}}$ uniformly for all $t>0$ and sufficiently large $n$. By the definition of $z_{n}$ and (10), we have

$$
\operatorname{Re} e^{z_{n}}=-\operatorname{Re} \frac{n+1}{z_{n}}=-\frac{n}{L}\left(1+O\left(\frac{L_{2}}{L}\right)\right)
$$

and

$$
\operatorname{Im} e^{z_{n}}=-\operatorname{Im} \frac{n+1}{z_{n}}=-\frac{\pi n}{L^{2}}\left(1+O\left(\frac{L^{2}}{L}\right)\right) .
$$

Using the inequality $0<\sqrt{x^{2}+y^{2}}-x \leq y^{2} /(2 x)$, which holds uniformly for all $x$ and $y$ with $0<y \leq x$, we obtain

$$
\left|\operatorname{Re} e^{z_{n}}+\right| e^{z_{n}}|| \leq \frac{1}{2}\left|\frac{\left(\operatorname{Im} e^{z_{n}}\right)^{2}}{\operatorname{Re} e^{z_{n}}}\right| \leq \frac{c_{2} n}{L^{3}},
$$

where $c_{2}$ is a positive constant. Therefore if $t$ is a real number satisfying $\operatorname{Re} w_{n} t<$ $-2 c_{2} / L^{2}$, i.e., $t>\left(4 c_{2} / \pi+o(1)\right) /\left(\left|w_{n}\right| L\right)$, then we have by $(11)$

$$
\begin{aligned}
\operatorname{Re}\left(e^{z_{n}}-e^{z_{n}+w_{n} t}\right) & \leq\left(\left|e^{z_{n}}\right|+\operatorname{Re} e^{z_{n}}\right)+\left(\left|e^{z_{n}}\right| e^{\operatorname{Re} w_{n} t}-\left|e^{z_{n}}\right|\right) \\
& \leq \frac{c_{2} n}{L^{3}}-\frac{2 c_{2}}{L^{2}}\left|e^{z_{n}}\right| \leq 0
\end{aligned}
$$

On the other hand, if $t$ is in the range $0<t \leq\left(4 c_{2} / \pi+o(1)\right) /\left(\left|w_{n}\right| L\right)$, then, by (10) and (11),

$$
\begin{aligned}
\operatorname{Re}\left(e^{z_{n}}-e^{z_{n}+w_{n} t}\right)= & \operatorname{Re}\left(-e^{z_{n}} w_{n} t\right)+O\left(\left|e^{z_{n}} w_{n}^{2}\right| t^{2}\right) \\
= & \operatorname{Re}\left\{\frac{n}{L}\left(1+\frac{L_{2}}{L}-\frac{\pi i}{L}+O\left(\frac{L_{2}}{L^{2}}\right)\right)\right. \\
& \left.\quad \sqrt{\frac{2 L}{n}}\left(-\frac{\pi}{2 L}+i+\frac{i L^{2}-i}{L}+O\left(\frac{L_{2}^{2}}{L^{2}}\right)\right) t\right\}+O\left(t^{2}\right) \\
= & \left(\frac{1}{\sqrt{2}}+o(1)\right) \sqrt{\frac{n}{L^{3}}} t+O\left(t^{2}\right) \leq c_{3} \sqrt{\frac{n}{L^{3}}} t
\end{aligned}
$$

for sufficiently large $n$, where $c_{3}$ is a positive constant. This proves the assertion that $\operatorname{Re} e^{z_{n}}-\operatorname{Re} e^{z_{n}+w_{n} t} \ll t \sqrt{n / L^{3}}$ uniformly for all $t>0$ and sufficiently large $n$.

We now estimate $I_{3}$. For $t$ in the interval $\left[1 /\left|w_{n}\right|^{1 / 3},\left|z_{n}\right| /\left|w_{n}\right|\right]$, the estimate (14) implies that

$$
\log \left|1+\frac{w_{n} t}{z_{n}}\right| \geq \frac{\left|w_{n}\right|}{4\left|z_{n}\right|} t
$$

for sufficiently large $n$. It follows that, by Lemma 1 ,

$$
\begin{aligned}
I_{3} & \leq \int_{1 /\left|w_{n}\right|^{1 / 3}}^{\left|z_{n} / w_{n}\right|} \exp \left\{c_{3} \sqrt{\frac{n}{L^{3}}} t-\frac{n}{4} \frac{\left|w_{n}\right|}{\left|z_{n}\right|} t\right\} d t \\
& \leq \frac{\left|z_{n}\right|}{\left|w_{n}\right|} \exp \left\{-\left(\frac{1}{4}+o(1)\right) \sqrt{\frac{n}{L}}\left|w_{n}\right|^{-1 / 3}\right\} \ll \exp \left\{-\frac{c_{4} n^{2 / 3}}{L^{2 / 3}}\right\}
\end{aligned}
$$


for some suitable positive constant $c_{4}$. The same bound holds for $I_{1}$. It remains to estimate $I_{2}$.

In the range $-1 /\left|w_{n}\right|^{1 / 3} \leq t \leq 1 /\left|w_{n}\right|^{1 / 3}$, we have, by Lemma 1 ,

$$
\begin{gathered}
\phi_{n}^{(3)}\left(z_{n}\right)=-e^{z_{n}}-\frac{2(n+1)}{z_{n}^{3}}=\frac{n+1}{z_{n}}+O\left(\frac{n}{L^{3}}\right) \ll \frac{n}{L}, \\
\phi_{n}^{(4)}\left(z_{n}+w_{n} t\right)=-e^{z_{n}+w_{n} t}+\frac{6(n+1)}{\left(z_{n}+w_{n}\right)^{3}} \ll \frac{n}{L} e^{\left|w_{n}\right|^{2 / 3}}+\frac{n}{L^{3}} \ll \frac{n}{L},
\end{gathered}
$$

and thus

$$
\begin{gathered}
\phi_{n}^{(3)}\left(z_{n}\right)\left(w_{n} t\right)^{3} \ll \frac{n}{L}\left|w_{n}\right|^{2} \ll 1, \\
\phi_{n}\left(z_{n}+w_{n} t\right)-\phi_{n}\left(z_{n}\right)-\phi_{n}^{\prime}\left(z_{n}\right) w_{n} t-\frac{\phi_{n}^{\prime \prime}\left(z_{n}\right)}{2}\left(w_{n} t\right)^{2}-\frac{\phi_{n}^{(3)}\left(z_{n}\right)}{6}\left(w_{n} t\right)^{3} \ll \frac{n}{L}\left|w_{n} t\right|^{4} \ll 1 .
\end{gathered}
$$

Since, by the definition of $z_{n}$ and $w_{n}, \phi_{n}^{\prime}\left(z_{n}\right)=0$ and $\phi_{n}^{\prime \prime}\left(z_{n}\right) w_{n}^{2} / 2=-1$, it follows that

$$
\begin{aligned}
I_{2} & =\int_{-1 /\left|w_{n}\right|^{1 / 3}}^{1 /\left|w_{n}\right|^{1 / 3}} \exp \left\{-t^{2}+\frac{\phi_{n}^{(3)}\left(z_{n}\right)}{6}\left(w_{n} t\right)^{3}+O\left(\frac{n}{L}\left|w_{n} t\right|^{4}\right)\right\} d t \\
& =\int_{-1 /\left|w_{n}\right|^{1 / 3}}^{1 /\left|w_{n}\right|^{1 / 3}} e^{-t^{2}}\left(1+\frac{\phi_{n}^{(3)}\left(z_{n}\right) w_{n}^{3}}{6} t^{3}+O\left(\left|\phi_{n}^{(3)}\left(z_{n}\right)^{2} w_{n}^{6}\right| t^{6}\right)+O\left(\frac{n\left|w_{n}\right|^{4}}{L} t^{4}\right)\right) d t \\
& =\sqrt{\pi}+O\left(\exp \left\{-\left|w_{n}\right|^{-2 / 3}\right\}\right)+O\left(\left|\phi_{n}^{(3)}\left(z_{n}\right)^{2} w_{n}^{6}\right|\right)+O\left(\frac{n\left|w_{n}\right|^{4}}{L}\right) \\
& =\sqrt{\pi}+O\left(\frac{L}{n}\right) .
\end{aligned}
$$

Combining this estimate, (15) and (16), we obtain

$$
\int_{\Gamma_{1}} \exp \left\{\phi_{n}(z)\right\} d z=w_{n} \exp \left\{\phi_{n}\left(z_{n}\right)\right\}\left(\sqrt{\pi}+O\left(\frac{L}{n}\right)\right) .
$$

Since $\int_{\Gamma_{2}}=-\bar{\int}_{\Gamma_{1}}$, it follows that

$$
\begin{aligned}
f(n) & =\frac{n ! e}{2 \pi i}\left(\int_{\Gamma_{1}}+\int_{\Gamma_{2}}\right)=\operatorname{Im} \frac{n ! e}{\sqrt{\pi}} w_{n} \exp \left\{\phi_{n}\left(z_{n}\right)\right\}+O\left(\frac{L}{n} n !\left|w_{n} \exp \left\{\phi_{n}\left(z_{n}\right)\right\}\right|\right) \\
& =\operatorname{Im} \Phi(n)+O\left(\frac{L}{n}|\Phi(n)|\right) .
\end{aligned}
$$

This completes the proof of Theorem 1. 


\section{Proofs of Corollaries}

Throughout this section, $L$ will denote $\log n$ or $\log t$, and $L_{2}$ will denote $\log \log n$ or $\log \log t$, depending on the context.

Proof of Corollary 1. By Theorem 1, we have

$$
|f(n)| \leq \frac{n ! e}{\sqrt{\pi}}\left|w_{n} \exp \left\{\phi_{n}\left(z_{n}\right)\right\}\right|\left(1+O\left(\frac{L}{n}\right)\right) .
$$

By Lemma 1 and the Stirling formula for $n$ !, it follows that

$$
\begin{aligned}
\log |f(n)| & \leq(n+1 / 2) \log n-n+\operatorname{Re} \phi_{n}\left(z_{n}\right)+O(1) \\
& =n\left(L-L_{2}-1+\frac{L_{2}+1}{L}+\frac{L^{2}-\pi^{2}}{2 L^{2}}+O\left(\frac{L_{2}^{3}}{L^{3}}\right)\right) .
\end{aligned}
$$

This proves Corollary 1. Corollary 2 is an immediate consequence of Corollary 1.

Proof of Corollary 3. We first note that the domains of the functions $z_{n}, w_{n}, \phi_{n}(z)$ and $\Phi(n)$ can be extended from the set of positive integers to the set of positive real numbers, and the asymptotic formulas in Lemma 1 remain valid with $n$ replaced by a positive real number $t$. From Theorem 1 we deduce that

$$
f(n)=|\Phi(n)|\left(\sin \theta(n)+O\left(\frac{L}{n}\right)\right),
$$

where

$$
\theta(t)=\operatorname{Im}\left(\phi_{t}\left(z_{t}\right)+\log w_{t}\right)
$$

By Lemma 1, we have

$$
\operatorname{Im} \log w_{t}=\frac{\pi}{2}+O\left(\frac{1}{L}\right)
$$

and

$$
\operatorname{Im} \phi_{t}\left(z_{t}\right)=-\frac{\pi t}{L}+O\left(\frac{t L_{2}}{L^{2}}\right)
$$

The claimed estimate (5) for $\theta(t)$ follows by inserting these estimates into (17).

We now prove estimate (6). By the definition of $z_{t}$, we have $z_{t} e^{z_{t}}+(t+1)=0$. Thus, the chain rule yields

$$
\frac{d z_{t}}{d t}=-\frac{1}{e^{z_{t}}\left(z_{t}+1\right)}=\frac{z_{t}}{(t+1)\left(z_{t}+1\right)}
$$

Since

$$
w_{t}^{2}=-\frac{2}{\phi_{t}^{\prime \prime}\left(z_{t}\right)}=-\frac{2}{-e^{z_{t}}+(t+1) / z_{t}^{2}}=-\frac{2}{(t+1) / z_{t}+(t+1) / z_{t}^{2}},
$$


by estimate (10) of Lemma 1 and (18), we have

$$
\frac{1}{w_{t}} \frac{d w_{t}}{d t}=-\frac{1}{2} \frac{\frac{d}{d t}\left(\frac{t+1}{z_{t}}+\frac{t+1}{z_{t}^{2}}\right)}{\left(\frac{t+1}{z_{t}}+\frac{t+1}{z_{t}^{2}}\right)}=-\frac{1}{2(t+1)}+\frac{z_{t}^{-2}+2 z_{t}^{-3}}{2\left(z_{t}^{-1}+z_{t}^{-2}\right)} \frac{d z_{t}}{d t} \ll \frac{1}{t} .
$$

Similarly, we have

$$
\begin{aligned}
\frac{d \phi_{t}\left(z_{t}\right)}{d t} & =\frac{d}{d t}\left(-e^{z_{t}}-(t+1) \log z_{t}\right) \\
& =-\log z_{t}-\left(e^{z_{t}}+\frac{t+1}{z_{t}}\right) \frac{d z_{t}}{d t}=-\log z_{t}
\end{aligned}
$$

and thus, by (10),

$$
\begin{aligned}
\operatorname{Im} \frac{d \phi_{t}\left(z_{t}\right)}{d t} & =-\operatorname{Im}\left\{\log \left(L-L_{2}+\pi i+\frac{L_{2}}{L}-\frac{\pi i}{L}+O\left(\frac{L_{2}^{2}}{L^{2}}\right)\right)\right\} \\
& =-\frac{\pi}{L}+O\left(\frac{L_{2}}{L^{2}}\right) .
\end{aligned}
$$

Combining this estimate and (19), we obtain

$$
\theta^{\prime}(t)=\operatorname{Im} \frac{1}{w_{t}} \frac{d w_{t}}{d t}+\operatorname{Im} \frac{d}{d t} \phi_{t}\left(z_{t}\right)=-\frac{\pi}{L}+O\left(\frac{L_{2}}{L^{2}}\right)
$$

This proves the estimate (6).

The proof of (7) is essentially the same as that of (6). By (18) and (20), we have

$$
\frac{d^{2}}{d t^{2}} \phi_{t}\left(z_{t}\right)=-\frac{d}{d t} \log z_{t}=-\frac{1}{z_{t}} \frac{z_{t}}{(t+1)\left(z_{t}+1\right)}=-\frac{1}{t\left(L-L_{2}+\pi i+1+O\left(L_{2} / L\right)\right)}
$$

and hence

$$
\operatorname{Im} \frac{d^{2}}{d t^{2}} \phi_{t}\left(z_{t}\right)=\frac{\pi}{t L^{2}}+O\left(\frac{L_{2}}{t L^{3}}\right) .
$$

Similarly, we have, by (18) and (19),

$$
\frac{d^{2}}{d t^{2}} \log w_{t}=\frac{1}{(t+1)^{2}}+\frac{1}{2} \frac{d}{d t}\left\{-\frac{z_{t}^{-2}+2 z_{t}^{-3}}{z_{t}^{-1}+z_{t}^{-2}} \frac{z_{t}}{(t+1)\left(z_{t}+1\right)}\right\} \ll \frac{1}{t^{2}} .
$$

Thus we conclude that

$$
\theta^{\prime \prime}(t)=\operatorname{Im} \frac{d^{2}}{d t^{2}} \phi_{t}\left(z_{t}\right)+\operatorname{Im} \frac{d^{2}}{d t^{2}} \log w_{t}=\frac{\pi}{t L^{2}}+O\left(\frac{L_{2}}{t L^{3}}\right) .
$$

This completes the proof of Corollary 3. 
Proof of Corollary 4. Let $\theta(t)$ be defined as in Corollary 3. Let $k$ be a positive integer, and let $t_{k}$ be the solution of $\theta(t)=-k \pi$. By Corollary $3, t_{k}$ satisfies

$$
k=\frac{t_{k}}{\log t_{k}}+O\left(\frac{t_{k} \log \log t_{k}}{\left(\log t_{k}\right)^{2}}\right)
$$

Hence, we obtain

$$
t_{k}=k \log t_{k}+O\left(\frac{t_{k} \log \log t_{k}}{\log t_{k}}\right)=k \log k+O(k \log \log k) .
$$

From this estimate we deduce that $n_{k}=t_{k}+O(1)$, and therefore estimate (8) holds.

To prove the second part of the corollary, we note that, by the mean value theorem, $\pi=\theta\left(t_{k}\right)-\theta\left(t_{k+1}\right)=\left(t_{k}-t_{k+1}\right) \theta^{\prime}\left(\xi_{k}\right)$, where $\xi_{k}$ is a real number between $t_{k}$ and $t_{k+1}$. The estimate (22) implies that

$$
\xi_{k}=t_{k}+O\left(t_{k+1}-t_{k}\right)=t_{k}+O(k \log \log k) .
$$

Hence, by (6) in Corollary 3, we have

$$
\begin{aligned}
n_{k+1}-n_{k} & =t_{k+1}-t_{k}+O(1)=-\frac{\pi}{\theta^{\prime}\left(\xi_{k}\right)} \\
& =\frac{\pi}{\frac{\pi}{\log t_{k}}+O\left(\frac{\log \log t_{k}}{\left(\log t_{k}\right)^{2}}\right)}=\log k+O(\log \log k),
\end{aligned}
$$

which is the claimed result.

\section{Proof of Theorem 2}

We will use the following well-known exponential sum estimate (see, e.g., [5, p. 17]).

Lemma 2 Let $a$ and $b$ be integers with $a<b$, and let $g$ be twice differentiable on $[a, b]$ with $g^{\prime \prime}(x) \geq \rho>0$ or $g^{\prime \prime}(x) \leq-\rho<0$ for some positive real number $\rho$ and all $x \in[a, b]$. Then

$$
\left|\sum_{n=a}^{b} e^{i g(n)}\right| \ll\left(\left|g^{\prime}(b)-g^{\prime}(a)\right|+1\right)\left(\rho^{-1 / 2}+1\right) .
$$

Proof of Theorem 2. It suffices to show that

$$
\left|\left\{x^{1 / 2}<n \leq x: f(n)=0\right\}\right| \ll x^{2 / 3} .
$$

In light of Corollary 3, we have for sufficiently large $x$

$$
\left|\left\{x^{1 / 2}<n \leq x: f(n)=0\right\}\right| \leq\left|\left\{x^{1 / 2}<n \leq x:\|\theta(n)\|<\frac{c_{1} \log n}{n}\right\}\right|
$$

THE ELECTRONIC JOURNAL OF COMBINATORICS 8 (2001), \#R19 
for some positive constant $c_{1}$, where $\theta(t)$ is the function occuring in the statement of Corollary 3 and $\|\theta(n)\|$ denotes the distance from $\theta(n)$ to the closest integer multiple of $\pi$. On the other hand, if $H=H(x)$ is an integer-valued function satisfying $H(x) \leq$ $\left(\pi /\left(2 c_{1}\right)\right) x^{1 / 2} / \log x$, then, for $x^{1 / 2}<n \leq x$, the condition $\|\theta(n)\|<c_{1} \log n / n$ implies that

$$
\left(\frac{\sin ((H+1) \theta(n))}{\sin \theta(n)}\right)^{2} \geq \frac{(H+1)^{2}}{\pi^{2}} .
$$

We therefore have

$$
\left|\left\{x^{1 / 2} \leq n \leq x: f(n)=0\right\}\right| \ll \frac{1}{H^{2}} \sum_{x^{1 / 2}<n \leq x}\left(\frac{\sin ((H+1) \theta(n))}{\sin \theta(n)}\right)^{2} .
$$

Using the identity

$$
\left(\frac{\sin ((H+1) t)}{\sin t}\right)^{2}=\sum_{h=-H}^{H}(H+1-|h|) e^{2 i h t}
$$

the right-hand side of (23) becomes

$$
\frac{1}{H^{2}} \sum_{h=-H}^{H}(H+1-|h|) S_{h} \leq \frac{x(H+1)}{H^{2}}+\frac{2}{H^{2}} \sum_{h=1}^{H}(H+1-h)\left|S_{h}\right|,
$$

where $S_{h}=\sum_{x^{1 / 2}<n \leq x} e^{2 i h \theta(n)}$.

We now estimate the exponential sum $S_{h}$. By Corollary 3, we have

$$
\left|h \theta^{\prime}(\lfloor x\rfloor)-h \theta^{\prime}\left(\left\lceil x^{1 / 2}\right\rceil\right)\right| \ll \frac{h}{\log x}
$$

and

$$
h \theta^{\prime \prime}(t) \gg \frac{h}{x(\log x)^{2}}
$$

for $x^{1 / 2}<t \leq x$. Thus, applying Lemma 2 with $g(t)=h \theta(t)$, we obtain

$$
\left|S_{h}\right| \ll\left(\frac{h}{\log x}+1\right)\left((\log x) \sqrt{\frac{x}{h}}+1\right) .
$$

Inserting this estimate in (24), we arrive at

$$
\begin{aligned}
\left|\left\{x^{1 / 2} \leq n \leq x: f(n)=0\right\}\right| & \ll \frac{x}{H}+\frac{1}{H} \sum_{h=1}^{H}\left(\sqrt{h x}+(\log x) \sqrt{\frac{x}{h}}+\frac{h}{\log x}+1\right) \\
& \ll \frac{x}{H}+\sqrt{H x}+(\log x) \sqrt{\frac{x}{H}}+\frac{H}{\log x}+1 .
\end{aligned}
$$

Taking $H=\left\lfloor x^{1 / 3}\right\rfloor$, we finally obtain

$$
\left|\left\{x^{1 / 2} \leq n \leq x: f(n)=0\right\}\right| \ll x^{2 / 3} .
$$

This completes the proof. 


\section{Acknowledgment}

The author would like to thank Professor Hildebrand of University of Illinois for helpful advice on the exposition of the paper. The author would also like to thank the referee for

thorough reading of the manuscript and for correcting a crucial mistake in the proof of Theorem 2.

\section{References}

[1] P. T. Bateman, Private communication.

[2] D. Bowman, Private communication.

[3] E. R. Canfield, P. Erdős and C. Pomerance, On a problem of Oppenheim concerning "Factorisatio Numerorum", J. Number Theory 17 (1983), 1-28.

[4] N. G. de Bruijn, Asymptotic methods in analysis, Corrected reprint of the third edition, Dover Publications, Inc., New York, 1981.

[5] L. Kuipers and H. Niederreiter, Uniform distribution of sequences, Pure and Applied Mathematics, Wiley-Interscience [John Wiley \& Sons], New York-LondonSydney, 1974.

[6] A. Oppenheim, On an arithmetic function, J. London Math. Soc. 1 (1926), 205211.

[7] M. V. Subbarao and A. Verma, Some remarks on a product expansion: an unexplored partition function, preprint.

[8] H. S. Wilf, Generatingfunctionology, Second Edition, Academic Press, Inc., Boston, 1994. 